\title{
Classification and fingerprinting of different berries based on biochemical profiling and antioxidant capacity
}

\author{
Jasminka Milivojević(1), Vera Rakonjac(1), Milica Fotirić Akšić(1), \\ Jelena Bogdanović Pristov ${ }^{(2)}$ and Vuk Maksimović(2)
}

\begin{abstract}
(1)University of Belgrade, Faculty of Agriculture, Nemanjina 6, 11080 Belgrade, Serbia. E-mail: jasminka@agrif.bg.ac.rs, verak@agrif.bg.ac.rs, fotiric@agrif.bg.ac.rs (2)University of Belgrade, Institute for Multidisciplinary Research, Kneza Višeslava 1a, 11000 Belgrade, Serbia. E-mail: mala@imsi.rs, maxivuk@imsi.rs
\end{abstract}

\begin{abstract}
The objective of this work was to evaluate the biochemical composition of six berry types belonging to Fragaria, Rubus, Vaccinium and Ribes genus. Fruit samples were collected in triplicate (50 fruit each) from 18 different species or cultivars of the mentioned genera, during three years (2008 to 2010). Content of individual sugars, organic acids, flavonols, and phenolic acids were determined by high performance liquid chromatography (HPLC) analysis, while total phenolics (TPC) and total antioxidant capacity (TAC), by using spectrophotometry. Principal component analysis (PCA) and hierarchical cluster analysis (CA) were performed to evaluate the differences in fruit biochemical profile. The highest contents of bioactive components were found in Ribes nigrum and in Fragaria vesca, Rubus plicatus, and Vaccinium myrtillus. PCA and CA were able to partially discriminate between berries on the basis of their biochemical composition. Individual and total sugars, myricetin, ellagic acid, TPC and TAC showed the highest impact on biochemical composition of the berry fruits. CA separated blackberry, raspberry, and blueberry as isolate groups, while classification of strawberry, black and red currant in a specific group has not occurred. There is a large variability both between and within the different types of berries. Metabolite fingerprinting of the evaluated berries showed unique biochemical profiles and specific combination of bioactive compound contents.
\end{abstract}

Index terms: bioactive compounds, metabolite fingerprinting, multivariate analysis, nutraceuticals, small fruits.

\section{Classificação e diversidade de pequenas frutas quanto ao perfil bioquímico e capacidade antioxidante}

Resumo - O objetivo deste trabalho foi avaliar a composição bioquímica de seis espécies de pequenas frutas dos gêneros Fragaria, Rubus, Vaccinium e Ribes. As amostras das frutas foram coletadas em triplicata (com 50 frutas cada) de 18 diferentes espécies ou cultivares dos gêneros mencionados, durante três anos (2008 a 2010). Os teores individuais de açúcares, ácidos orgânicos, flavonóis e ácidos fenólicos foram determinados por cromatografia líquida de alta performance (HPLC), e os fenóis totais (FT) e a capacidade antioxidante total (CAT), por meio de espectrofotometria. As análises de componente principal (ACP) e de agrupamento hierárquico $(\mathrm{AH})$ foram utilizadas para avaliar as diferenças no perfil bioquímico das frutas. Os maiores teores de compostos bioativos foram encontrados em Ribes nigrum e em Fragaria vesca, Rubus plicatus e Vaccinium myrtillus. ACP e AH foram capazes de discriminar parcialmente as frutas com base em sua composição bioquímica. Os acúcares totais e individuais, a miricetina, o ácido elágico, FT e CAT foram os parâmetros que mais impactaram na avaliação da composição bioquímica das pequenas frutas. AH fez a separação de amora-preta, framboesa e mirtilo como grupos isolados, porém a classificação do morango, da groselha-preta e da groselha-vermelha, em um grupo específico, não ocorreu. Há grande variabilidade entre e dentro dos diferentes tipos de frutas vermelhas. A identificação de metabólitos das pequenas frutas mostrou perfis bioquímicos únicos e combinações específicas de teores de compostos bioativos.

Termos par indexação: compostos bioativos, identificação de metabólitos, análise multivariada, nutracêuticos, pequenas frutas.

\section{Introduction}

Berries are widely recognized for their nutritional quality and potential health benefits. Recent increasing interest in nutraceuticals and functional foods has led plant breeders to initiate selection of crops such as strawberries (Tulipani et al., 2008), raspberries (Fotirić Akšić et al., 2011; Mikulić-Petkovšek et al., 2012), blackberries (Kafkas et al., 2006), blueberries (Remberg et al., 2007), bilberries (Šavikin et al., 2009), 
and currants (Milivojević et al., 2012) with higher contents of taste- and health-related compounds.

Considering genetic differences among wild relatives from natural population and cultivars of berries, a potential variability can be observed in their contents and composition of bioactive compounds (Remberg et al., 2007). Berries are rich sources of phytochemicals such as sugars, organic acids, and phenolics. Sugars and organic acids are their main soluble constituents and have major effect on taste and fruit ripeness, or even represent a suitable index of consumer's acceptability (Kafkas et al., 2006; Tosun et al., 2009). Type and quantity of individual compounds also affect fruit taste; therefore, the composition and concentration of these compounds may reflect changes in fruit quality (Kafkas et al., 2006).

An important question that arises from the preliminary screening of berries relates to the polyphenolic profiles of the species, specifically, which components within these profiles contribute most to differences in bioactive potential. These compounds are mainly represented by flavonoids, phenolic acids, and tannins, which are known as natural antioxidants (Määttä-Riihinen et al., 2004). Ellagic acid is the predominant phenolic acid in strawberries, raspberries and blackberries (Koponen et al., 2007), and chlorogenic acid in blueberries (Zheng \& Wang, 2003). Apart from phenolic acids, these berries have been shown to contain high levels of flavonols, such as quercetin, kaempferol, and myricetin, as well as their derivatives (primarily glycosides), which may provide health benefits as dietary antioxidants (Siriwoharn et al., 2004). Combining this information with an increasing knowledge of the structure of these compounds has enabled the selection of parents for breeding new, highly health-protective cultivars for the future.

Since computational methods were introduced for the metabolic fingerprinting methodology, a new analytical level has been reached. Principal component analysis (PCA) and cluster analysis (CA) (Terry et al., 2005; Sarbu et al., 2012) can help to classify individual samples according to their metabolite composition. Correlation among variables is common in this type of biological data, which can simplify the characterization of berry fruits (Badenes et al., 2000).

The objective of this work was to evaluate the biochemical composition of six berry types belonging to Fragaria, Rubus, Vaccinium and Ribes genus.

\section{Materials and Methods}

Eighteen different types of berry were used in this study. Samples included different species, cultivars and accessions from native population (Table 1). Berries of Fragaria vesca L., Rubus idaeus L., Rubus plicatus L., and Vaccinium myrtillus L. were harvested from native populations in Western Serbia (municipality of Dragačevo), and compared to ripe fruits of two strawberry cultivars of $F$. ananassa Duch. ('Marmolada' and 'Madeleine'), two raspberry cultivars ('Willamette' and 'Meeker'), two thornless blackberry cultivars ('Thornfree' and 'Čačanska bestrna'), and two high bush blueberry cultivars ('Berkeley', and 'Bluecrop'). Additionally, three black currant cultivars ('Ben Lomond', 'Ben Sarek' and 'Malling Juel') and three red currant cultivars ('Heinemann's Rote Spatleze', 'Rondom' and 'Red Versailles') were analyzed. The study was conducted during three consecutive years (2008-2010) in an experimental field established in the same region where native fruit were harvested $\left(43^{\circ} 46^{\prime} \mathrm{N}, 20^{\circ} 12^{\prime} \mathrm{E}, 350 \mathrm{~m}\right.$ altitude).

The region is characterized by moderate continental climate. The average temperature during the growing seasons was $16.3^{\circ} \mathrm{C}$, approximately $2^{\circ} \mathrm{C}$ higher than the long-term average (1980-2007). Precipitation in the growing seasons was different in the investigated years. The highest level of $997.5 \mathrm{~mm}$ was recorded in 2010, when it surpassed the long-year average by $25 \%$.

Fruit samples were collected in triplicate (50 fruit each). Only berries at the commercial maturity stage were harvested. Approximately $100 \mathrm{~g}$ of fruit from each replicate were pureed, and samples of $5 \mathrm{~g}$ were homogenized for $1 \mathrm{~min}$ in $20 \mathrm{~mL}$ extraction solution containing methanol/water/hydrochloric acid (70:30:5 ratio by volume). The homogenate was filtered using a filter paper, and the filtrates were centrifuged at 3,000 $\mathrm{g}$ for $15 \mathrm{~min}$. Methanol supernatant was divided into aliquots, and frozen at $-80^{\circ} \mathrm{C}$ until analysis.

Analyses of sugars were performed on a chromatographic system Waters Breeze, (Waters, Milford, MA, USA) containing 1,525 binary pumps system, a thermostated column compartment, and the Waters 2465 electrochemical detector, equipped with gold working electrodes and hydrogen-referent electrodes. Separation was performed on CarboPac PA1 (Dionex, Sunnyvale, CA, USA) 250x4 mm column equipped with corresponding CarboPac PA1 guard column. Sugars were eluted with $200 \mathrm{mmol} \mathrm{L}^{-1} \mathrm{NaOH}$ 
for $20 \mathrm{~min}$, at $1.0 \mathrm{~mL} \mathrm{~min}{ }^{-1}$ flow rate at $30^{\circ} \mathrm{C}$ constant temperature. Signals were detected in a pulse mode with the following waveform: $\mathrm{E}_{1}=+0.1 \mathrm{~V}$, for $280 \mathrm{~ms}$; $E_{2}=+0.75 \mathrm{~V}$, for $150 \mathrm{~ms} ; \mathrm{E}_{3}=-0.85 \mathrm{~V}$, for $150 \mathrm{~ms}$ and within $80 \mathrm{~ms}$ of integration time. Filter timescale was $0.2 \mathrm{~s}$, and range was 200 to $500 \mathrm{nA}$ for the full $\mathrm{mV}$ scale. Data acquisition and evaluation were carried out by Waters Empower 2 software (Waters, Milford, MA, USA).

A HP 1100 photo diode array detector, HP1100 series, (Hewlett Packard, Palo Alto, CA, USA) was adjusted to $210 \mathrm{~nm}$, with a reference signal at $600 \mathrm{~nm}$, in order to analyze organic acids. An anion exchange column Aminex HPX-87H, (Bio-Rad Lab, CA, USA) $300 \times 7.8 \mathrm{~mm}$ was used with $5 \mathrm{mmol} \mathrm{L}^{-1} \mathrm{H}_{2} \mathrm{SO}_{4}$ as a mobile phase. The elution was isocratic, with $0.6 \mathrm{~mL} \mathrm{~min}^{-1}$ flow rate at $30^{\circ} \mathrm{C}$. Data acquisition and evaluation was done by Agilent Chemstation software (Agilent Chemstation, Palo Alto, CA, USA).

Ascorbic acid was measured by a Merck RQflex reflectometer (Merck KGaA, Darmstadt, Germany) as described by Pantelidis et al. (2007). Results were expressed as milligrams of ascorbic acid per 100 grams of fresh weight (mg $100 \mathrm{~g}^{-1}$ ).

Quantification of individual phenolic compounds was done by reversed phase HPLC analysis. Samples were injected in a Waters HPLC system consisting of 1,525 binary pumps, thermostat, and 717+ autosampler connected to a Waters 2996 diode array detector (Waters,
Milford, MA, USA). Chromatograms were gathered in 3D mode with extracted signals at specific wavelengths for different compounds $(370,326$, and $254 \mathrm{~nm}$, respectively). Separation of phenolics was performed on a Symmetry C-18 RP column $(125 \times 4 \mathrm{~mm})$ with a $5 \mu \mathrm{m}$ particle diameter (Waters, Milford, MA, USA) connected to the appropriate guard column. With the following gradient profile, two mobile phases, A ( $0.1 \%$ phosphoric acid) and B (acetonitrile), were used at $1 \mathrm{~mL} \mathrm{~min}{ }^{-1}$ flow: first 20 min from $10-22 \%$ B, next 20 min of linear rise up to $40 \%$ B, followed by $5 \mathrm{~min}$ reverse to $10 \% \mathrm{~B}$, and an additional $7.5 \mathrm{~min}$ of equilibration time. Data acquisition and spectral evaluation for peak confirmation were done with Waters Empower 2 Software (Waters, Milford, MA, USA).

The method employed for determination of total phenolic content (TPC) was based on Folin-Ciocalteu phenol reagent and spectrophotometric determination (Singleton \& Rossi Junior, 1965). Results were expressed as milligrams of gallic-acid equivalent per gram of fresh weight $\left(\mathrm{mg} \mathrm{g}^{-1}\right)$.

Total antioxidant capacity (TAC) was measured by 2,2'azino-bis-3-ethylbenzothiazoline-6-sulfonic acid (ABTS) method according to Arnao et al. (1999). Results were expressed as milligrams of ascorbic acid equivalent per gram of fresh weight $\left(\mathrm{mg} \mathrm{g}^{-1}\right)$.

Analysis of variance was performed to evaluate the influence of genotypes on chemical composition of fruits, and the least significant difference (LSD) test

Table 1. Analyzed species and cultivars of different berries, their origin, and parentage.

\begin{tabular}{|c|c|c|c|}
\hline Species & Cultivar & Origin & Pedigree \\
\hline \multirow{2}{*}{ Fragaria ananassa Duch. } & Marmolada & Italy & Sel.15 x Gorella \\
\hline & Madeleine & Italy & Addie x Miranda \\
\hline Fragaria vesca $\mathrm{L}$. & / & Serbia & Wild accession \\
\hline \multirow{2}{*}{ Rubus idaeus L. } & Willamette & USA & Newburgh x Lloyd George \\
\hline & Meeker & USA & Willamette x Cuthbert \\
\hline R. idaeus subsp. vulgatus Arrhen. & l & Serbia & Wild accession \\
\hline \multirow{2}{*}{ Rubus sp. } & Thornfree & USA & US1410 (Brainerd x Merton Thornless) x US1414 (Merton Thornless x Eldorado) \\
\hline & Čačanska bestrna & Serbia & Dirksen Thornless x Black Saten \\
\hline Rubus plicatus L. & l & Serbia & Wild accession \\
\hline \multirow{2}{*}{ Ribes rubrum L. } & Heinemann's Rote Spatleze & Germany & Red Dutch x (Alwin Lorgus x Red Dutch) \\
\hline & Red Versailles & France & Unknown \\
\hline \multirow[t]{2}{*}{ Ribes multiflorum L. } & Rondom & Netherlands & Backcross of R. multiflorum \\
\hline & Ben Lomond & Scotland & (Consort x Magnus) x (Brödtorp x Janslunda) \\
\hline \multirow[t]{2}{*}{ Ribes nigrum L. } & Ben Sarek & Scotland & Goliath x Öjebyn \\
\hline & Malling Juel & England & Unknown \\
\hline \multirow{2}{*}{ Vaccinium corymbosum $\mathrm{L}$. } & Bluecrop & USA & GM 37 (Jersey x Pioneer) x CU 5 (Stanley x June) \\
\hline & Berkeley & USA & Stanley x GS 149 (Jersey x Pioneer) \\
\hline Vaccinium myrtillus L. & l & Serbia & Wild accession \\
\hline
\end{tabular}


was used to compare the means, at 5\% probability. To identify the patterns of multi-trait variation in berry fruit collection, principal component analysis (PCA) and cluster analysis (CA) were applied. Average values from three-year measurements were use in the statistical analyses. CA was performed by Ward's method using Euclidean distances. Pearson's correlation coefficient between traits was determined. Data analysis was made using the Statistica for Windows software, version 5.0 (StatSoft Inc., Tulsa, OK, USA) statistical package.

\section{Results and Discussion}

Fructose was the predominant sugar in all studied types of berry (species and cultivars), followed by glucose (Table 2). Monosacharides were present in large amounts compared with the sucrose portion, probably due to high invertase activity during the final maturation stages. For strawberries, the highest average contents of glucose, fructose and sucrose were obtained in $F$. vesca. In raspberries, significant differences were observed for fructose and total sugar content (Table 2). The total sugar content corresponds to the sum of glucose, fructose and sucrose reported by Wang et al. (2008) for blueberries grown in a conventional culture system.

Citric and malic acids are the main organic acids in berry fruit species (Basson et al., 2010). In this study (Table 2), the main organic acid was citric acid, while twice as low quantities of malic acid were observed in all tested strawberries and blueberries. Raspberries and red currants showed citric acid quantities three times higher than malic acid, while in black currants

Table 2. Contents ${ }^{(1)}$ of sugars, organic acids, and ascorbic acid of berry fruits ${ }^{(2)}$.

\begin{tabular}{|c|c|c|c|c|c|c|c|c|}
\hline \multirow[t]{2}{*}{ Genotype } & Glucose & Fructose & Sucrose & Total sugars & Citric acid & Malic acid & Total acids & Ascorbic acid \\
\hline & \multicolumn{8}{|c|}{----------------------------- $\left(\mathrm{mg} \mathrm{g}^{-1}\right.$ ) -------------------------- } \\
\hline & \multicolumn{8}{|c|}{ Strawberry } \\
\hline Fragaria ananassa 'Marmolada' & $45.2 b$ & $58.8 \mathrm{~b}$ & $7.4 \mathrm{~b}$ & $111.4 \mathrm{~b}$ & $0.072 b$ & $0.026 \mathrm{~b}$ & $0.097 \mathrm{~b}$ & $78.0 \mathrm{~b}$ \\
\hline Fragaria ananassa 'Madeleine' & $45.8 \mathrm{~b}$ & $62.1 b$ & $3.7 \mathrm{c}$ & $111.6 b$ & $0.080 \mathrm{~b}$ & $0.023 b$ & $0.103 b$ & $96.6 \mathrm{a}$ \\
\hline Fragaria vesca & $65.2 \mathrm{a}$ & $116.8 \mathrm{a}$ & $27.4 \mathrm{a}$ & $209.4 \mathrm{a}$ & $0.207 \mathrm{a}$ & $0.105 \mathrm{a}$ & $0.313 \mathrm{a}$ & $97.2 \mathrm{a}$ \\
\hline \multirow[t]{2}{*}{ Mean } & $52.0 \mathrm{~B}$ & $79.2 \mathrm{C}$ & $12.9 \mathrm{~A}$ & $144.1 \mathrm{~B}$ & $0.120 \mathrm{DE}$ & $0.051 \mathrm{C}$ & $0.171 \mathrm{E}$ & $90.6 \mathrm{~B}$ \\
\hline & \multicolumn{8}{|c|}{ Raspberry } \\
\hline Rubus idaeus 'Willamette' & 35.2 & $46.8 \mathrm{a}$ & 6.5 & $88.5 \mathrm{ab}$ & $0.243 \mathrm{a}$ & $0.054 \mathrm{~b}$ & $0.297 \mathrm{a}$ & $40.9 b$ \\
\hline R. idaeus 'Meeker' & 36.2 & $49.2 \mathrm{a}$ & 5.3 & $90.7 \mathrm{a}$ & $0.181 b$ & $0.043 b$ & $0.223 b$ & $44.3 b$ \\
\hline R. idaeus & 38.3 & $31.5 b$ & 7.0 & $76.8 \mathrm{~b}$ & $0.146 \mathrm{c}$ & $0.080 \mathrm{a}$ & $0.227 \mathrm{~b}$ & $56.1 \mathrm{a}$ \\
\hline \multirow[t]{2}{*}{ Mean } & $36.6 \mathrm{C}$ & $42.5 \mathrm{D}$ & $6.2 \mathrm{~B}$ & $85.3 \mathrm{C}$ & $0.190 \mathrm{C}$ & $0.059 \mathrm{C}$ & $0.249 \mathrm{C}$ & $47.1 \mathrm{D}$ \\
\hline & \multicolumn{8}{|c|}{ Blackberry } \\
\hline Rubus sp. 'Thornfree' & 67.7 & $88.1 \mathrm{a}$ & 3.01 & $158.9 \mathrm{a}$ & 0.101 & $0.171 \mathrm{a}$ & $0.272 \mathrm{a}$ & $79.0 \mathrm{~b}$ \\
\hline Rubus sp. 'Čačanska bestrna' & 66.8 & $86.0 \mathrm{a}$ & 4.2 & $157.1 \mathrm{a}$ & 0.088 & $0.116 b$ & $0.204 b$ & $68.8 \mathrm{c}$ \\
\hline R. plicatus & 64.5 & $76.1 \mathrm{~b}$ & 3.0 & $143.6 \mathrm{~b}$ & 0.097 & $0.031 \mathrm{c}$ & $0.128 \mathrm{c}$ & $104.9 \mathrm{a}$ \\
\hline \multirow[t]{2}{*}{ Mean } & $66.3 \mathrm{~A}$ & $83.4 \mathrm{~B}$ & $3.4 \mathrm{C}$ & $153.2 \mathrm{~A}$ & $0.095 \mathrm{E}$ & $0.106 \mathrm{~A}$ & $0.201 \mathrm{DE}$ & $84.2 \mathrm{~B}$ \\
\hline & \multicolumn{8}{|c|}{ Red currant } \\
\hline Ribes rubrum 'Heinneman'ş Rote Spatleze' & $81.1 \mathrm{a}$ & $113.1 \mathrm{a}$ & $14.5 \mathrm{a}$ & $208.7 \mathrm{a}$ & $0.323 \mathrm{a}$ & $0.131 \mathrm{a}$ & $0.456 \mathrm{a}$ & $52.9 \mathrm{c}$ \\
\hline R. rubrum 'Red Versailles' & $55.9 \mathrm{~b}$ & $82.7 b$ & $4.1 b$ & $142.7 b$ & $0.235 b$ & $0.042 b$ & $0.280 \mathrm{~b}$ & $96.8 \mathrm{a}$ \\
\hline R. multiflorum 'Rondom' & $50.3 \mathrm{c}$ & $76.2 \mathrm{~b}$ & $4.0 \mathrm{~b}$ & $130.4 b$ & $0.223 b$ & $0.060 \mathrm{~b}$ & $0.283 b$ & $72.8 \mathrm{~b}$ \\
\hline \multirow[t]{2}{*}{ Mean } & $62.5 \mathrm{~A}$ & $90.7 \mathrm{~A}$ & $7.5 \mathrm{~B}$ & $160.6 \mathrm{~A}$ & $0.260 \mathrm{~B}$ & $0.078 \mathrm{~B}$ & $0.340 \mathrm{~B}$ & $74.2 \mathrm{C}$ \\
\hline & \multicolumn{8}{|c|}{ Black currant } \\
\hline Ribes nigrum 'Ben Lomond' & $78.8 \mathrm{a}$ & $117.6 \mathrm{a}$ & $10.1 \mathrm{a}$ & $206.6 \mathrm{a}$ & $0.544 b$ & $0.132 \mathrm{a}$ & $0.677 \mathrm{~b}$ & $118.2 \mathrm{c}$ \\
\hline R. nigrum 'Ben Sarek' & $52.3 \mathrm{~b}$ & $73.3 \mathrm{c}$ & $6.5 b$ & $132.1 \mathrm{c}$ & $0.685 \mathrm{a}$ & $0.140 \mathrm{a}$ & $0.826 \mathrm{a}$ & $132.6 b$ \\
\hline R. nigrum 'Malling Juel' & $57.7 \mathrm{~b}$ & $84.1 \mathrm{~b}$ & $3.4 \mathrm{c}$ & $145.2 b$ & $0.339 \mathrm{c}$ & $0.049 \mathrm{~b}$ & $0.390 \mathrm{c}$ & $141.4 \mathrm{a}$ \\
\hline \multirow[t]{2}{*}{ Mean } & $62.9 \mathrm{~A}$ & $91.7 \mathrm{~A}$ & $6.7 \mathrm{~B}$ & $161.3 \mathrm{~A}$ & $0.523 \mathrm{~A}$ & $0.107 \mathrm{~A}$ & $0.631 \mathrm{~A}$ & $130.7 \mathrm{~A}$ \\
\hline & \multicolumn{8}{|c|}{ Blueberry } \\
\hline Vaccinium corymbosum 'Berkeley' & $70.8 \mathrm{a}$ & 88.7 & $5.2 \mathrm{c}$ & 164.7 & $0.096 b$ & $0.067 \mathrm{~b}$ & $0.163 b$ & $9.7 b$ \\
\hline V. corymbosum 'Bluecrop' & $64.4 b$ & 84.4 & $7.6 b$ & 156.4 & $0.110 \mathrm{~b}$ & $0.048 \mathrm{~b}$ & $0.158 b$ & $8.1 \mathrm{~b}$ \\
\hline V. myrtillus & $57.8 \mathrm{c}$ & 87.1 & $10.3 \mathrm{a}$ & 155.2 & $0.234 \mathrm{a}$ & $0.116 \mathrm{a}$ & $0.350 \mathrm{a}$ & $25.8 \mathrm{a}$ \\
\hline Mean & $64.3 \mathrm{~A}$ & $86.7 \mathrm{AB}$ & $7.7 \mathrm{~B}$ & $158.8 \mathrm{~A}$ & $0.147 \mathrm{D}$ & $0.077 \mathrm{~B}$ & $0.224 \mathrm{CD}$ & $14.5 \mathrm{E}$ \\
\hline
\end{tabular}


this ratio was 5:1. However, malic acid was found to be dominant in blackberries. Kafkas et al. (2006) reported similar amounts of malic acids, but they did not detect citric acid in blackberry cultivars.

Berries, particularly black currant cultivars, are a rich source of ascorbic acid (Milivojević et al., 2012). The highest level of ascorbic acid was measured in 'Malling Juel'. Although the ascorbic acid content was on average 1.5 to 3 fold lower in strawberries, raspberries, blackberries and red currants, compared to black currants, their average values over $40 \mathrm{mg}$ per $100 \mathrm{~g}$ of fresh weight are still high, and make them a rich source of vitamin C.

Differences were not always significant when comparing the content of sugars, organic acids, and vitamin $\mathrm{C}$ between wild berry species and cultivars. Various factors may influence their biochemical composition, such as genotype, growth conditions including environmental factors, and cultivation techniques (Scalzo et al., 2005).

Great variability existed, however, among the examined berries regarding content of individual flavonols, in which black currants stand out (Table 3). The highest average content of myricetin was detected in black currant 'Ben Sarek' and $V$. myrtillus, while the highest values of quercetin was observed in black currants, wild strawberry and blueberry 'Bluecrop' (over $4 \mu \mathrm{g} \mathrm{g}^{-1} \mathrm{FW}$ ).

Significant differences in free ellagic acid were observed between all types of berries, in which strawberry

Table 3. Contents ${ }^{(1)}$ of flavonols, phenolic acids and total phenolics (TPC), and total antioxidant capacity (TAC) of berry fruits ${ }^{(2)}$.

\begin{tabular}{|c|c|c|c|c|c|c|c|}
\hline \multirow[t]{2}{*}{ Genotype } & Myricetin & Kaempferol & Quercetin & Ellagic acid & Chlorogenic acid & TPC & TAC \\
\hline & & & $\left(\mu \mathrm{g} \mathrm{g}^{-1}\right)$ & & -- & \multicolumn{2}{|c|}{----- $\left(\mathrm{mg} \mathrm{g}^{-1}\right)$------ } \\
\hline & \multicolumn{7}{|c|}{ Strawberry } \\
\hline Fragaria ananassa 'Marmolada' & $0.50 \mathrm{~b}$ & $2.35 \mathrm{~b}$ & $1.79 \mathrm{~b}$ & $21.3 \mathrm{c}$ & 0.0 & $1.09 \mathrm{~b}$ & $1.48 \mathrm{~b}$ \\
\hline Fragaria ananassa 'Madeleine' & $1.04 \mathrm{ab}$ & $2.89 \mathrm{a}$ & $1.60 \mathrm{~b}$ & $32.2 \mathrm{~b}$ & 0.0 & $0.92 b$ & $1.50 \mathrm{~b}$ \\
\hline Fragaria vesca & $1.25 \mathrm{a}$ & $2.35 \mathrm{~b}$ & $7.34 \mathrm{a}$ & $122.5 \mathrm{a}$ & 0.0 & $4.69 \mathrm{a}$ & $5.78 \mathrm{a}$ \\
\hline \multirow[t]{2}{*}{ Mean } & $0.93 \mathrm{D}$ & $2.53 \mathrm{~B}$ & $3.58 \mathrm{~B}$ & $58.7 \mathrm{~A}$ & 0.0 & $2.23 \mathrm{C}$ & $2.92 \mathrm{BC}$ \\
\hline & \multicolumn{7}{|c|}{ Raspberry } \\
\hline Rubus idaeus 'Willamette' & 0.26 & $2.40 \mathrm{a}$ & $0.71 \mathrm{a}$ & $2.0 \mathrm{c}$ & 0.0 & $2.22 \mathrm{a}$ & 3.13 \\
\hline R. idaeus 'Meeker' & 0.31 & $0.40 \mathrm{~b}$ & $0.37 \mathrm{~b}$ & $5.2 \mathrm{~b}$ & 0.0 & $1.02 \mathrm{~b}$ & 1.32 \\
\hline R. idaeus & 0.25 & $0.13 b$ & $0.82 \mathrm{a}$ & $12.7 \mathrm{a}$ & 0.0 & $1.10 \mathrm{~b}$ & 1.41 \\
\hline \multirow[t]{2}{*}{ Mean } & $0.27 \mathrm{E}$ & $0.98 \mathrm{D}$ & $0.63 \mathrm{~A}$ & $6.6 \mathrm{D}$ & 0.0 & $1.45 \mathrm{D}$ & $1.95 \mathrm{CD}$ \\
\hline & \multicolumn{7}{|c|}{ Blackberry } \\
\hline Rubus sp. 'Thornfree' & $1.02 \mathrm{~b}$ & $1.88 \mathrm{~b}$ & 0.30 & $35.4 \mathrm{~b}$ & 0.0 & $1.97 \mathrm{~b}$ & $2.47 \mathrm{~b}$ \\
\hline Rubus sp. 'Čačanska bestrna' & $0.31 \mathrm{~b}$ & $1.54 \mathrm{~b}$ & 0.42 & $30.9 \mathrm{c}$ & 0.0 & $1.74 \mathrm{~b}$ & $2.45 \mathrm{~b}$ \\
\hline R. plicatus & $3.14 \mathrm{a}$ & $2.76 \mathrm{a}$ & 0.45 & $61.7 \mathrm{a}$ & 0.0 & $3.20 \mathrm{a}$ & $4.95 \mathrm{a}$ \\
\hline \multirow[t]{2}{*}{ Mean } & $1.49 \mathrm{C}$ & $2.06 \mathrm{C}$ & $0.39 \mathrm{E}$ & $42.7 \mathrm{~B}$ & 0.0 & $2.30 \mathrm{C}$ & $3.29 \mathrm{BC}$ \\
\hline & \multicolumn{7}{|c|}{ Red currant } \\
\hline Ribes rubrum 'Heinneman'ş Rote Spatleze' & $0.84 \mathrm{ab}$ & $0.73 b$ & $2.54 \mathrm{~b}$ & $3.0 \mathrm{~b}$ & 0.0 & 1.69 & 2.13 \\
\hline R. rubrum 'Red Versailles' & $1.28 \mathrm{a}$ & $1.22 \mathrm{a}$ & $3.14 \mathrm{a}$ & $6.0 \mathrm{a}$ & 0.0 & 0.81 & 0.95 \\
\hline R. multiflorum 'Rondom' & $0.46 \mathrm{~b}$ & $1.39 \mathrm{a}$ & $2.00 \mathrm{c}$ & $4.9 \mathrm{ab}$ & 0.0 & 1.29 & 1.21 \\
\hline \multirow[t]{2}{*}{ Mean } & $0.86 \mathrm{D}$ & $1.11 \mathrm{D}$ & $2.56 \mathrm{C}$ & $4.7 \mathrm{E}$ & 0.0 & $1.27 \mathrm{E}$ & $1.43 \mathrm{D}$ \\
\hline & \multicolumn{7}{|c|}{ Black currant } \\
\hline Ribes nigrum 'Ben Lomond' & $5.56 \mathrm{~b}$ & $1.06 \mathrm{~b}$ & $6.78 \mathrm{a}$ & $12.9 \mathrm{a}$ & 0.0 & $4.71 \mathrm{a}$ & $7.60 \mathrm{a}$ \\
\hline R. nigrum 'Ben Sarek' & $7.23 \mathrm{a}$ & $1.29 \mathrm{ab}$ & $4.20 \mathrm{c}$ & $5.7 \mathrm{c}$ & 0.0 & $2.46 \mathrm{~b}$ & $3.83 \mathrm{~b}$ \\
\hline R. nigrum 'Malling Juel' & $5.46 \mathrm{~b}$ & $1.60 \mathrm{a}$ & $4.88 \mathrm{~b}$ & $9.9 \mathrm{~b}$ & 0.0 & $3.85 \mathrm{a}$ & $5.68 \mathrm{ab}$ \\
\hline \multirow[t]{2}{*}{ Mean } & $6.08 \mathrm{~A}$ & $1.32 \mathrm{D}$ & $5.29 \mathrm{~A}$ & $9.5 \mathrm{C}$ & 0.0 & $3.67 \mathrm{~A}$ & $5.70 \mathrm{~A}$ \\
\hline & \multicolumn{7}{|c|}{ Blueberry } \\
\hline Vaccinium corymbosum 'Berkeley' & $1.72 b$ & $1.47 \mathrm{c}$ & $3.73 b$ & $6.1 \mathrm{~b}$ & $41.0 \mathrm{~b}$ & $1.50 \mathrm{~b}$ & $2.51 \mathrm{~b}$ \\
\hline V. corymbosum 'Bluecrop' & $1.76 \mathrm{~b}$ & $4.75 \mathrm{a}$ & $4.76 \mathrm{a}$ & $19.1 \mathrm{a}$ & $71.2 \mathrm{a}$ & $1.99 \mathrm{~b}$ & $2.45 \mathrm{~b}$ \\
\hline V. myrtillus & $10.67 \mathrm{a}$ & $3.04 \mathrm{~b}$ & $2.78 \mathrm{c}$ & $8.0 \mathrm{~b}$ & $20.0 \mathrm{c}$ & $3.87 \mathrm{a}$ & $6.16 \mathrm{a}$ \\
\hline Mean & $4.72 \mathrm{~B}$ & $3.09 \mathrm{~A}$ & $3.75 \mathrm{~B}$ & $11.1 \mathrm{C}$ & 44.1 & $2.45 \mathrm{~B}$ & $3.71 \mathrm{~B}$ \\
\hline
\end{tabular}


showed the highest values. Among strawberries, F. vesca is the richest source of this component. In accordance with earlier findings for ellagic acid content in Georgia-grown blackberries (Sellappan et al., 2002), significant quantities of this acid were also detected in the analyzed blackberry cultivars, which showed values twice as low as that recorded in $R$. plicatus (wild accession). This discrepancy is most likely due to genetic differences, since fruit of $R$. plicatus were taken from native populations in Western Serbia, whereas the studied cultivars were derived from hybridization. Chlorogenic acid, which contributes to the tart taste of fruit and fruit products, was present only in blueberries. Vaccinium myrtillus had the lowest chlorogenic acid content, whereas higher quantities were detected in the blueberry cultivars, as previously reported by Kalt et al. (2007).

The total phenolic contents (TPC) recorded in the wild accession $F$. vesca, $V$. myrtillus, and $R$. plicatus were higher than those obtained for the corresponding cultivars (Table 3 ). Besides black currants, the highest total antioxidant capacities (TAC) were also recorded in V. myrtillus, F. vesca, and R. plicatus. Such results confirm the need to evaluate the diversity of native populations of these species and, based on this, well-focused breeding programs can create new cultivars specifically selected for improved antioxidant potential.

In this study, all variables related to sugars (Table 4) were correlated with each other $(r=0.523-0.990)$. These results seem to be quite expectable, since quantities of monosaccharide directly influence the amount of total sugars. The only exception was the absence of correlation between sucrose and glucose content, which coincides with the results reported by Drogoudi et al. (2008), for apricot. Both citric and malic acids were significantly correlated to total acid content $(r=0.978$ and 0.632 , respectively), but did not correlate to each other, which was also previously stated by Zheng et al. (2009). Ascorbic acid contents did not correlate with most of the studied variables, contrarily to what was previously reported by Ramaiya et al. (2013). These authors, however, studied passion fruit in an absolutely diverse climatic condition than the ones observed in the present study. Ascorbic acid was significantly correlated with citric acid $(\mathrm{r}=0.509)$ and chlorogenic acid $(r=-0.649)$. In the group of phenolics, none of the components correlated with each other, indicating that those traits are genetically independent. A positive correlation was obtained between TPC and sugars $(r=0.514-0.595)$, which agrees with the results of Abidi et al. (2011). The only exception was the absence of the relationship between TPC and glucose content. A good correlation between sugar content and levels of phenolic substances in berries are due to the role of sugars in the regulation of phenolic biosynthesis (Pirie \& Mullins, 1977). Significant correlation between quercetin and myricetin, on one side, and with TPC, on the other, suggest that those two flavonols represent the most important components of TPC. Positive correlation was also observed between both TAC and fructose, and TAC and total sugars. The ascorbic acid content did not contribute to the antioxidant activity of berries, as shown by the weak correlation observed here and by Luximon-Ramma et al. (2003).

Table 4. Correlation matrix among biochemical compounds studied in berry fruits ${ }^{(1)}$.

\begin{tabular}{|c|c|c|c|c|c|c|c|c|c|c|c|c|c|c|}
\hline Compound & $\mathrm{F}$ & $\mathrm{S}$ & TS & $\mathrm{CA}$ & MA & TA & $\mathrm{AA}$ & M & $\mathrm{K}$ & Q & EA & $\mathrm{CHA}$ & TPC & $\mathrm{TAC}^{(2)}$ \\
\hline Glucose & 0.906 & 0.293 & 0.934 & 0.162 & 0.530 & 0.268 & 0.066 & 0.213 & 0.079 & 0.459 & 0.217 & 0.235 & 0.440 & 0.433 \\
\hline Fructose $(\mathrm{F})$ & & 0.523 & 0.990 & 0.294 & 0.514 & 0.379 & 0.182 & 0.288 & 0.109 & 0.666 & 0.345 & 0.124 & 0.595 & 0.547 \\
\hline Sucrose (S) & & & 0.561 & 0.166 & 0.315 & 0.220 & 0.027 & 0.052 & 0.058 & 0.601 & 0.610 & 0.008 & 0.514 & 0.392 \\
\hline Total sugars (TS) & & & & 0.257 & 0.537 & 0.352 & 0.132 & 0.245 & 0.098 & 0.647 & 0.373 & 0.153 & 0.581 & 0.531 \\
\hline Citric acid CA & & & & & 0.454 & 0.978 & 0.509 & 0.573 & 0.331 & 0.513 & 0.260 & 0.241 & 0.436 & 0.475 \\
\hline Malic acid (MA) & & & & & & 0.632 & 0.093 & 0.325 & 0.262 & 0.190 & 0.062 & 0.139 & 0.369 & 0.350 \\
\hline Total acids (TA) & & & & & & & 0.466 & 0.575 & 0.351 & 0.493 & 0.211 & 0.243 & 0.467 & 0.496 \\
\hline Ascorbic acid (AA) & & & & & & & & 0.235 & 0.240 & 0.275 & 0.251 & 0.649 & 0.357 & 0.359 \\
\hline Myricetin (M) & & & & & & & & & 0.173 & 0.401 & 0.140 & 0.097 & 0.629 & 0.719 \\
\hline Kaempferol (K) & & & & & & & & & & 0.149 & 0.296 & 0.602 & 0.205 & 0.184 \\
\hline Quercetin (Q) & & & & & & & & & & & 0.280 & 0.267 & 0.651 & 0.606 \\
\hline Ellagic acid (EA) & & & & & & & & & & & & 0.122 & 0.448 & 0.325 \\
\hline Chlorogenic acid (CHA) & & & & & & & & & & & & & 0.035 & 0.028 \\
\hline Total phenolic content (TPC) & & & & & & & & & & & & & & 0.978 \\
\hline
\end{tabular}

${ }^{(1)}$ Correlation coefficient values higher than 0.496 are significant at $5 \%$ probability. ${ }^{(2)} \mathrm{TAC}$, total antioxidant capacity. 
The strong correlation between TPC and TAC suggests that the antioxidant activity of berries is derived mainly from the content of phenolic compounds in fruits, which agrees with Ehala et al. (2005).

Four principal components (PC), with eigenvalues higher than 1, were obtained from principal component analysis (Table 5). Variables with correlation coefficient larger than 0.70 (absolute value) were determined only in PC1. Those were glucose, fructose, total sugars, ellagic acid, total phenolics and TAC. The highest contribution of PC2 (over 0.60 of absolute value) corresponded to variables such as malic acid, myricetin, quercetin and ascorbic acid content. High loading on PC3 (over 0.60 of absolute value) had chlorogenic and ascorbic acid. Finally, the largest scores of PC4 were due to total acids content.

The first component was mainly related to variation of total sugars, TPC and TAC (Figure 1). By this component, genotypes with relatively high content were located on the right side, such as black currant 'Ben Lomond' and $F$. vesca wild accession. Strawberry genotypes 'Marmolada' and 'Madeleine', and three raspberry genotypes were on the left side: 'Willamette' and 'Meeker', as well as $R$. idaeus wild accession, which have relatively low content of these components.

The distribution along the second component was mainly caused by malic acid, myricetin, quercetin and ascorbic acid content. Quercetin and ascorbic acid were

Table 5. Principal component loadings of the variables.

\begin{tabular}{lcccc}
\hline Variable & PC1 & PC2 & PC3 & PC4 \\
\hline Glucose & $0.707^{*}$ & 0.344 & 0.011 & 0.479 \\
Fructose & $0.848^{*}$ & 0.311 & 0.088 & 0.313 \\
Sucrose & 0.586 & 0.323 & 0.376 & 0.029 \\
Total sugars & $0.838^{*}$ & 0.353 & 0.110 & 0.352 \\
Citric acid & 0.394 & 0.541 & 0.406 & 0.338 \\
Malic acid & 0.627 & 0.668 & 0.232 & 0.006 \\
Total acids & 0.612 & 0.210 & 0.021 & 0.487 \\
Ascorbic acid & 0.013 & 0.664 & 0.673 & 0.088 \\
Myricetin & 0.691 & 0.631 & 0.196 & 0.121 \\
Kaempferol & 0.596 & 0.248 & 0.545 & 0.331 \\
Quercetin & 0.039 & 0.686 & 0.338 & 0.447 \\
Ellagic acid & $0.786^{*}$ & 0.159 & 0.100 & 0.141 \\
Chlorogenic acid & 0.338 & 0.481 & 0.666 & 0.343 \\
Total phenolic content & $0.855^{*}$ & 0.065 & 0.003 & 0.407 \\
Total antioxidant content & $0.831^{*}$ & 0.010 & 0.109 & 0.413 \\
\hline Eigenvalue & 6.1753 & 2.86 & 1.74 & 1.61 \\
Variance (\%) & 41.2 & 19.0 & 11.6 & 10.7 \\
Cumulative (\%) & 41.2 & 60.2 & 71.8 & 82.6 \\
\hline
\end{tabular}

*Loadings higher than 0.700 . positively connected to $\mathrm{PC} 2$, whereas malic acid and myricetin negatively. In this regard, blueberry 'Bluecrop', which has a low content of ascorbic acid and myricetin, stood at the positive site of this PC2, but black currant 'Ben Sarek', which is characterized by high contents of vitamin $\mathrm{C}$ and myricetin, stood at the negative site.

A grouping of genotypes on scatter plot shows that the separation was done according to the types of berries (Figure 1). The two strawberry cultivars almost completely overlapped, and the wild accession was found in the exactly opposite side of the scatter plot. This can be explained by the fact that the cultivars belong to octaploid $(2 \mathrm{n}=8 \mathrm{x}=56)$ species $F$. ananassa, while the wild accession belongs to the diploid $(2 \mathrm{n}=2 \mathrm{x}=14)$ species $F$. vesca. Therefore, with increasing ploidy, level content of bioactive components decreased. Raspberries on one side, and blackberry genotypes, on the other, are part of a homogeneous group, suggesting that these two types of berry fruits have a unique profile for the content of bioactive components, regardless of whether they are cultivars or wild relatives. Grouping of red currant was such that 'Rondom' and 'Red Versailles' were separated as a unique group, while 'Heinneman's Rote Spatleze' was located on the opposite side. All cultivars of black currant were found in the same square of the scatter plot. According to their position, 'Ben Sarek' and 'Malling Juel' can be classified in one group, while 'Ben Lomond' is independent. Two blueberry cultivars were classified in the same group, while the wild accession $V$. myrtillus distinguished as an independent genotype. Previously, this analysis was applied to biochemical profiling of many berries (Bordonaba \& Terry, 2008; Kim et al., 2009; Gorinstein et al., 2013), and good results for the discrimination of species or cultivars were also obtained.

Red currant 'Heinneman's Rote Spatleze' and black currant 'Ben Lomond', together with strawberry $F$. vesca and blueberry $V$. myrtillus wild accessions, were separated from the other genotypes of the same groups. Discrimination of these four genotypes indicated that they have very high contents of some compounds. In that regard, 'Heinneman's Rote Spatleze' has high total sugar content (Table 2), whereas 'Ben Lomond' has high content of total sugar, myricetin, and TPC, and the highest TAC (Table 3). Blueberry was primarily characterized by a high myricetin content and TAC. The wild strawberry accession $F$. vesca showed the best biochemical profile, which was distinguished by a 
high content of total sugars (Table 2), quercetin, ellagic acid, TPC, and TAC (Table 3). Therefore, these genotypes can be recommended as the best sources of health-related compounds for fresh consumption and processing.

Similar groupings were obtained using the CA, by which the 18 berry genotypes were clustered into six main groups (Figure 2). Although the number of

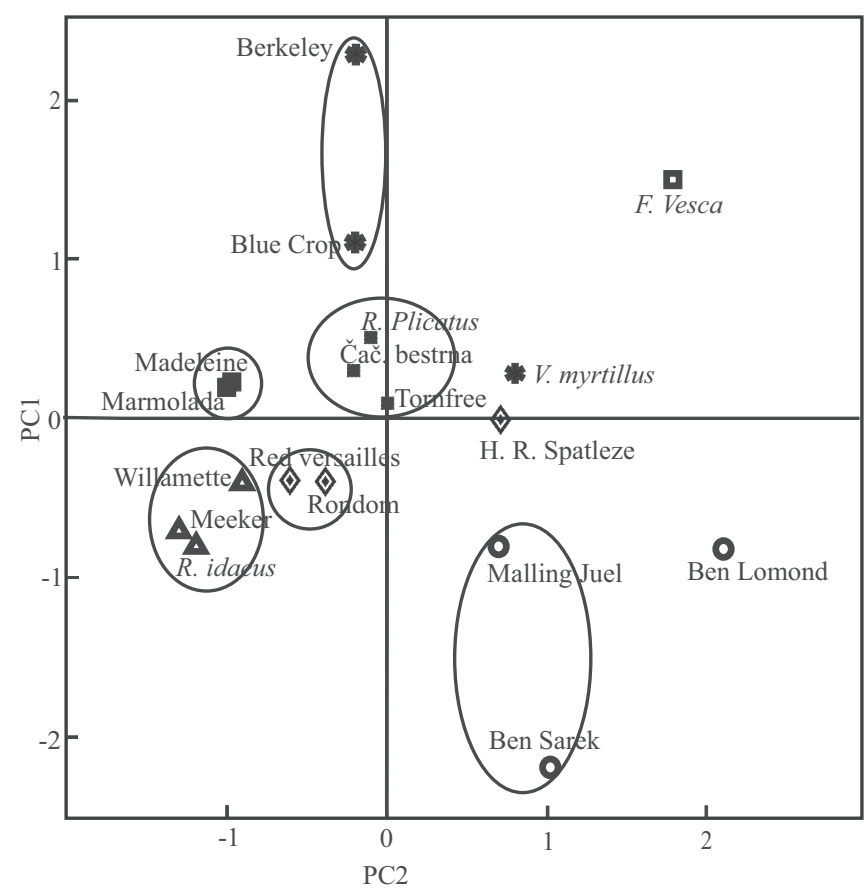

Figure 1. Scatter plot for $\mathrm{PC} 1$ and $\mathrm{PC} 2$, obtained by biochemical profiles of 18 diverse genotypes representing six different berry fruit types: strawberry ( $\boldsymbol{\square})$, raspberry $(\triangle)$, blackberry $(\square)$, red currant $(\bullet)$, black currant $(\bigcirc)$, and blueberry (*).

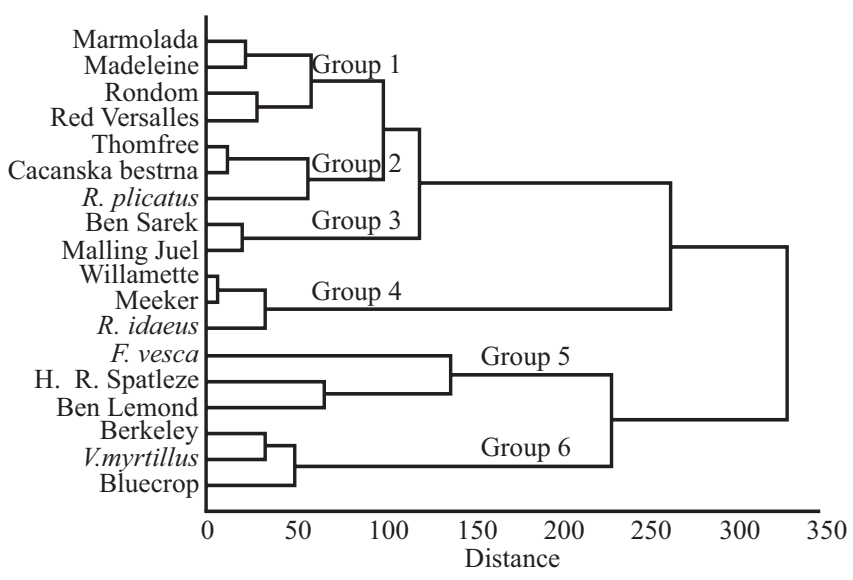

Figure 2. Hierarchical analysis dendrogram obtained by Ward's method using 15 biochemical compounds of berry fruits. clusters corresponds to the number of berry fruit types, separation according to the type was not fully shown. On the obtained dendrogram, blackberries (group II), raspberries (group IV) and blueberries (group VI) were established as a separate group. Clustering of strawberry, and red and black currant into separate groups was not achieved. Thus, two strawberry cultivars ('Marmolada' and 'Madeleine') and two red currant cultivars ('Rondom' and 'Red Versailles') were classified in the same group (group I). Black currant 'Ben Sarek' and 'Malling Juel' formed group III. Greatest heterogeneity was observed in group $\mathrm{V}$, in which wild strawberry accession, red currant 'Heinneman's Rote Spatleze', and black currant 'Ben Lomond' are located.

\section{Conclusions}

1. Metabolite fingerprinting of Fragaria, Rubus, Vaccinium, and Ribes wild and cultivated berries shows that most of the biochemical compounds differ between and within the studied berry types.

2. Total phenolic content followed a pattern similar to total antioxidant capacity in all tested samples.

3. A large number of metabolite contents are correlated, which allows for the use of a smaller number of variables without losing much information.

4. For characterizing and classifying the types berries, individual and total sugars, myricetin, ellagic acid, total phenolic content, and total antioxidant capacity have the highest influence.

5. The best composition of bioactive components is found in the cultivars of black currant (Ribes nigrum), as well as in Fragaria vesca, Rubus plicatus and Vaccinium myrtillus.

\section{Acknowledgements}

To the Serbian Ministry of Education, Science and Technological Development (projects III46008, III43010, 173040, and 173017), for financial support.

\section{References}

ABIDI, W.; JIMĖNEZ, S.; MORENO, M.Á.; GOGORCENA, Y. Evaluation of antioxidant compounds and total sugar content in a nectarine [Prunus persica (L.) Batsch] progeny. International Journal of Molecular Sciences, v.12, p.6919-6935, 2011. DOI: 10.3390/ijms12106919.

ARNAO, M.B.; CANO, A.; ACOSTA, M. Methods to measure the antioxidant activity in plant material. A comparative 
discussion. Free Radical Research, v.31, p.89-96, 1999. DOI: 10.1080/10715769900301371.

BADENES, M.L.; MARTINEZ-CALVO, J.; LLÁCER, G. Analysis of a germplasm collection of loquat (Eriobotrya japonica Lindl.). Euphytica, v.114, p.187-194, 2000. DOI: 10.1023/A:1003950215426.

BASSON, C.E.; GROENEWALD, J.-H.; KOSSMANN, J.; CRONJE, C.; BAUER, R. Sugar and acid-related quality attributes and enzyme activities in strawberry fruits: invertase is the main sucrose hydrolysing enzyme. Food Chemistry, v.121, p.1156-1162, 2010. DOI: 10.1016/j.foodchem.2010.01.064.

BORDONABA, J.G.; TERRY, L.A. Biochemical profiling and chemometric analysis of seventeen UK-grown black currant cultivars. Journal of Agricultural and Food Chemistry, v.56, p.7422-7430, 2008. DOI: 10.1021/jf8009377.

DROGOUDI, P.D.; VEMMOS, S.; PANTELIDIS, G.; PETRI, E.; TZOUTZOUKOU, C.; KARAYIANNIS, I. Physical characters and antioxidant, sugar, and mineral nutrient contents in fruit from 29 apricot (Prunus armeniaca L.) cultivars and hybrids. Journal of Agricultural and Food Chemistry, v.56, p.10754-10760, 2008. DOI: $10.1021 /$ jf801995x.

EHALA, S.; VAHER, M.; KALJURAND, M. Characterization of phenolic profiles of Northern European berries by capillary electrophoresis and determination of their antioxidant activity. Journal of Agricultural and Food Chemistry, v.53, p.6484-6490, 2005. DOI: $10.1021 / \mathrm{jf050397w.}$

FOTIRIĆ AKŠIĆ, M.; RADOVIĆ, A.; MILIVOJEVIĆ, J.; NIKOLIĆ, M.; NIKOLIĆ, D. Genetic parameters of yield components and pomologic properties in raspberry seedlings. Genetika, v.43, p.667-674, 2011. DOI: 10.2298/GENSR1103667F.

GORINSTEIN, S.; ARANCIBIA-AVILA, P.; TOLEDO, F.; NAMIESNIK, J.; LEONTOWICZ, H.; LEONTOWICZ, M.; HAM, K.S.; KANG, S.G., VEARASIL, K.; SUHAJ, M. Application of analytical methods for the determination of bioactive compounds in some berries. Food Analytical Methods, v.6, p.432-444, 2013. DOI: $10.1007 / \mathrm{s} 12161-012-9453-\mathrm{z}$.

KAFKAS, E.; KOSAR, M.; TÜREMIS, N.; BASER, K.H.C. Analysis of sugars, organic acids and vitamin $\mathrm{C}$ contents of blackberry genotypes from Turkey. Food Chemistry, v.97, p.732-736, 2006. DOI: 10.1016/j.foodchem.2005.09.023.

KALT, W.; JOSEPH, J.A.; SHUKITT-HALE, B. Blueberries and human health: a review of current research. Journal of the American Pomological Society, v.61, p.151-160, 2007.

KIM, S.W.; MIN, S.R.; KIM, J.; PARK, S.K.; KIM, T.I.; LIU, J.R. Rapid discrimination of commercial strawberry cultivars using Fourier transform infrared spectroscopy data combined by multivariate analysis. Plant Biotechnology Report, v.3, p.87-93, 2009. DOI: 10.1007/s11816-008-0078-z.

KOPONEN, J.M.; HAPPONEN, A.M.; MATTILA, P.H.; TÖRRÖNEN, A.R. Contents of anthocyanins and ellagitannins in selected foods consumed in Finland. Journal of Agricultural and Food Chemistry, v.55, p.1612-1619, 2007. DOI: 10.1021/ jf062897a.

LUXIMON-RAMMA, A.; BAHORUN, T.; CROZIER, A. Antioxidant actions and phenolic and vitamin $\mathrm{C}$ contents of common Mauritian exotic fruits. Journal of the Science of Food and Agriculture, v.83, p.496-502, 2003. DOI: 10.1002/jsfa.1365.

MÄÄTTÄ-RIIHINEN, K.R.; KAMAL-ELDIN, A.; TÖRRÖNEN, A.R. Identification and quantification of phenolic compounds in berries of Fragaria and Rubus species (family Rosaceae). Journal of Agricultural and Food Chemistry, v.52, p.6178-6187, 2004. DOI: $10.1021 /$ jf049450r.

MIKULIČ-PETKOVŠEK, M.; SCHMITZER, V.; SLATNAR, A.; ŠTAMPAR, F.; VEBERIČ, R. Composition of sugars, organic acids, and total phenolics in 25 wild or cultivated berry species. Journal of Food Science, v.77, p.1064-1070, 2012. DOI: 10.1111/j.1750-3 841.2012.02896.x.

MILIVOJEVIĆ, J.; SLATNAR, A.; MIKULIČ-PETKOVŠEK, M.; ŠTAMPAR, F.; NIKOLIĆ, M.; VEBERIČ, R. The influence of early yield on the accumulation of major taste and health related compounds in black and red currant cultivars (Ribes spp.). Journal of Agricultural and Food Chemistry, v.60, p.2682-2691, 2012. DOI: $10.1021 / \mathrm{jf} 204627 \mathrm{~m}$.

PANTELIDIS, G.E.; VASILAKAKIS, M.; MANGANARIS, G.A.; DIAMANTIDIS, G. Antioxidant capacity, phenol, anthocyanin and ascorbic acid contents in raspberries, blackberries, red currants, gooseberries and Cornelian cherries. Food Chemistry, v.102, p.777-783, 2007. DOI: 10.1016/j.foodchem.2006.06.021.

PIRIE, A.; MULLINS, M.G. Interrelationships of sugars, anthocyanins, total phenols and dry weight in the skin of grape berries during ripening. American Journal of Enology and Viticulture, v.28, p.204-209, 1977.

RAMAIYA, S.D.; BUJANG, J.S.; ZAKARIA, M.H.; KINGA, W.S.; SAHRIRA, M.A.S. Sugars, ascorbic acid, total phenolic content and total antioxidant activity in passion fruit (Passiflora) cultivars. Journal of the Science of Food and Agriculture, v.93, p.1198-205, 2013. DOI: 10.1002/jsfa.5876.

REMBERG, S.F.; MÅGE, F.; HAFFNER, K.; BLOMHOFF, R. Highbush blueberries Vaccinium corymbosum L., raspberries Rubus idaeus L. and black currants Ribes nigrum L. - influence of cultivar on antioxidant activity and other quality parameters. Acta Horticulturae, v.744, p.259-266, 2007.

SARBU, C.; NASCU-BRICIU, R.D.; KOT-WASIK, A.; GORINSTEIN, S.; WASIK, A.; NAMIESNIK, J. Classification and fingerprinting of kiwi and pomelo fruits by multivariate analysis of chromatographic and spectroscopic data. Food Chemistry, v.130, p.994-1002, 2012. DOI: 10.1016/j.foodchem.2011.07.120.

ŠAVIKIN, K.; ZDUMIĆ, G.; JANKOVIĆ, T.; TASIĆ, S.; MENKOVIĆ, N.; STEVIĆ, T.; DJORDJEVIĆ, B. Phenolic content and radical scavenging capacity of berries and related jams from certificated area in Serbia. Plant Foods for Human Nutrition, v.64, p.212-217, 2009. DOI: 10.1007/s11130-009-0123-2.

SCALZO, J.; POLITI, A.; PELlEGRINI, N.; MEZZETI, B.; BATTINO, M. Plant genotype affects total antioxidant capacity and phenolic contents in fruit. Nutrition, v.2, p.207-213, 2005. DOI: 10.1016/j.nut.2004.03.025.

SELLAPPAN, S.; AKOH, C.C.; KREWER, G. Phenolic compounds and antioxidant capacity of Georgia-grown blueberries and blackberries. Journal of Agricultural and Food Chemistry, v.50, p.2432-2438, 2002. DOI: 10.1021/jf011097r.

SINGLETON, V.L.; ROSSI JUNIOR, J.A. Colorimetry of total phenolics with phosphomolybdic-phosphotungstic acid reagents. 
American Journal of Enology and Viticulture, v.16, p.144-158, 1965.

SIRIWOHARN, T.; WROLSTAD, R.E.; FINN, C.E; PEREIRA, C.B. Influence of cultivar, maturity, and sampling on blackberry (Rubus L. hybrids) anthocyanins, polyphenolics, and antioxidant properties. Journal of Agricultural and Food Chemistry, v.52, p.8021-8030, 2004. DOI: 10.1021/jf048619y.

TERRY, L.A.; WHITE, S.F.; TIGWELL, L.J. The application of biosensors to fresh produce and the wider food industry. Journal of Agricultural and Food Chemistry, v.53, p.1309-1316, 2005. DOI: $10.1021 /$ jf040319t.

TOSUN, M.; ERCISLI, S.; KARLIDAG, H.; SENGUL, M. Characterization of red raspberry (Rubus idaeus L.) genotypes for their physicochemical properties. Journal of Food Science, v.74, p.575-579, 2009. DOI: 10.1111/j.1750-3841.2009.01297.x.

TULIPANI, S.; MEZZETTI, B.; CAPOCASA, F.; BOMPADRE, S.; BEEKWILDER, J.; RIC DEVOS, H.C.; CAPANOGLU, E.;
BOVY, A.; BATTINO, M. Antioxidants, phenolic compounds, and nutritional quality of different strawberry genotypes. Journal of Agricultural and Food Chemistry, v.56, p.696-704, 2008. DOI: 10.1021/jf0719959.

WANG, S.Y.; CHEN, C.-T.; SCIARAPPA, W.; WANG, C.Y.; CAMP, M.J. Fruit quality, antioxidant capacity, and flavonoid content of organically and conventionally grown blueberries. Journal of Agricultural and Food Chemistry, v.56, p.5788-5794, 2008. DOI: $10.1021 /$ jf703775r.

ZHENG, J.; BAORU, Y.; TUOMASJUKKA, S.; SHIYI, O.; KALLIO, H. Effects of latitude and weather conditions on contents of sugars, fruit acids, and ascorbic acid in black currant (Ribes nigrum L.) juice. Journal of Agricultural and Food Chemistry, v.57, p.2977-2987, 2009. DOI: 10.1021/jf8034513.

ZHENG, W.; WANG, S.Y. Oxygen radical absorbing capacity of phenolics in blueberries, cranberries, chokeberries, and lingonberries. Journal of Agricultural and Food Chemistry, v.51, p.502-509, 2003. DOI: 10.1021/jf020728u.

Received on April 16, 2013 and accepted on August 30, 2013 